\title{
PRELIMINARY RESULTS OF RADIO-ASTRONOMICAL OBSERVATIONS OF ANNULAR SOLAR ECLIPSE, APRIL 19, $195^{8}$
}

\author{
A. P. MOLChaNOV \\ Academy of Sciences, Moscow, U.S.S.R. \\ CHEN FAN-YUN \\ Institute of Radio and Electronics, Academia Sinica, Peking, C.P.R. \\ WANG SHOU-KUANG \\ Peking Observatory, Peking, C.P.R. \\ E. G. MIRZABEKIAN AND A. E. SALOMONOVICH \\ Academy of Sciences, Moscow, US.S.R.
}

Radio-astronomical observations of the annular eclipse of 1958 April 19 $\left(\eta=r_{e} / r_{\odot}=0.939, \varphi=18^{\circ} 14^{\prime} 34^{\prime \prime} .08, \lambda=-7^{\mathrm{h}} 18^{\mathrm{m}} 01^{\mathrm{s}} 86\right)$ were especially interesting since this year was, apparently, the year of maximum solar activity. Some preliminary results of the observations carried out by a joint expedition of the Soviet and Chinese Academies of Sciences, equipped with seven radio telescopes, are described in this report.*

Most of the observational units $(\lambda=3.2,4.5,5.1 \mathrm{~cm})$ registered distinct changes in the derivative of antenna-temperature variation at the moments of covering and uncovering of sunspots by the lunar disk. The greatest radio-frequency radiation source, which consisted of two regions, was caused by a great sunspot group near the center of the disk.

Angular dimensions of radiation regions were found to be close to those of sunspots and the percentage of sunspot flux in the total solar radio emission was as follows: for $3.2 \mathrm{~cm}, 6$ per cent; for $4.5 \mathrm{~cm}, 11.5$ per cent; for $5.1 \mathrm{~cm}, 16.5$ per cent.

The regions of increased steepness were distinctly registered at 4.5 and $5.1 \mathrm{~cm}$ wavelengths near third and fourth contacts. The examination of records permitted the supposition that besides the observed increase of brightness at the solar limb there was a radio-frequency radiation region above the spot located at this moment 23 degrees behind the solar limb. ${ }^{\dagger}$

The radiation of a local source caused by a great sunspot group was also measured at $\lambda=3.2 \mathrm{~cm}$ by using a method based on the determination of the displacement of the effective solar center during the eclipse.

When the group was uncovered the effective center acquired an additional

* The expedition was prepared under Professor Khaikin's leadership.

t There is a possibility of ascribing this source to the coronal region having a bright green line, which is symmetrical with the sunspot relative equatorial plane. 
2.5 minutes of arc displacement toward the group. The average effective temperature of the whole group has been determined from the known distance between the source and the effective center of radiation. It was found to be $1,600,000^{\circ} \mathrm{K}$.

The polarized component of solar radiation was observed at wavelengths of $0.8,2.0,3.3,5.1$, and $50 \mathrm{~cm}$. This component, however, was not detected at $\lambda=0.8 \mathrm{~cm}$ or at $\lambda=2.0 \mathrm{~cm} .^{*}$

The region of origin of circularly polarized component at $\lambda=3.3$ and $\lambda=5.1 \mathrm{~cm}$ corresponded to two regions above the great sunspot group referred to.

The antenna temperature at the middle point between the second and third contacts was measured by all units registering nonpolarized radiation flux. It was found to be 17 per cent (relative to total sun value) for $\lambda=0.8 \mathrm{~cm}$; 21 per cent for $\lambda=3.2,4.5$, and $5.1 \mathrm{~cm}$; and 25 per cent for $\lambda=50 \mathrm{~cm}$. The uncovered part of the solar surface was 11.8 per cent. These results of eclipse-curve analysis may be explained better by the rise of solar brightness at the solar limb than by the considerable increase of the solar diameter.

Two radiation minima were distinctly registered at $4.5-$ and $5.1-\mathrm{cm}$ wavelengths. Their depths were found to be different. These minima and the steep parts of the curves near first and fourth contacts bear witness also to the supposed existence of a bright solar limb.

The greater depth of the second minimum and the greater value of the derivative of the second region of increased steepness may be sufficiently explained by the above-mentioned radiation source on the edge of the solar disk.

The records may be used to compute the distribution of radio brightness over the solar disk if the distribution is assumed to have circular symmetry.

Such an assumption is justified by the solar-ellipticity measurements carried out during the eclipse at $3.2-\mathrm{cm}$ wavelength. Ellipticity was determined by measuring the second harmonic of the scanning by the direction-finding technique used earlier to investigate the displacement of the sun's effective center. An evaluation of the results has shown that the radiation-intensity ratio between equatorial regions and the poles did not exceed 1.1.

It should be noted in conclusion that the successful work of this expedition was possible thanks to joint efforts of the Academy of Sciences of the U.S.S.R. and the Academy of Sciences of the Chinese People's Republic, and to the great assistance rendered by various organizations of the Chinese People's Republic.

* The more detailed results of polarized component observations are treated separately. 